\title{
ATLASGAL, the APEX Telescope Large Area Survey of the Galaxy
}

\author{
F. Schuller ${ }^{1}$, K. M. Menten ${ }^{1}$, F. Wyrowski ${ }^{1}$, \\ H. Beuther ${ }^{2}$, S. Bontemps ${ }^{3}$, L. Bronfman ${ }^{4}$, Y. Contreras ${ }^{4}$, \\ T. Henning ${ }^{2}$, F. Motte ${ }^{5}$, P. Schilke ${ }^{6}$, M. Walmsley ${ }^{7}$ and A. Zavagno ${ }^{8}$ \\ ${ }^{1}$ MPIfR, Bonn, Germany; email: schuller@mpifr-bonn.mpg.de \\ ${ }^{2}$ MPIA, Heidelberg, Germany; ${ }^{3}$ LAB, Bordeaux, France; ${ }^{4}$ Univ. de Chile, Santiago, Chile \\ ${ }^{5} \mathrm{CEA} / \mathrm{DSM} / \mathrm{IRFU} / \mathrm{SAp}$, Saclay, France; ${ }^{6}$ Universität zu Köln, Germany \\ ${ }^{7}$ Osservatorio Astrofisico di Arcetri, Italy; ${ }^{8} \mathrm{LAM}$, Marseille, France
}

\begin{abstract}
Submillimeter continuum emission traces high molecular column densities and, thus, dense cloud regions in which new stars are forming. Surveys of the Galactic plane in such emission have the potential of delivering an unbiased view of high-mass star formation throughout the Milky Way. Here we present the scope, current status and first results of ATLASGAL, an ongoing survey of the Galactic plane using the Large APEX Bolometer Camera (LABOCA) on the Atacama Pathfinder Experiment (APEX) telescope at the Chajnantor plateau in Chile. Aimed at mapping 360 square degrees at $870 \mu \mathrm{m}$, with a uniform sensitivity of $50 \mathrm{mJy} / \mathrm{beam}$, this survey will provide the first unbiased sample of cold dusty clumps in the Galaxy at submillimeter wavelengths. These will be targets for molecular line follow-up observations and high resolution studies with ALMA and the EVLA.
\end{abstract}

Keywords. Surveys, submillimeter, stars: formation, ISM: structure, Galaxy: disk

The ATLASGAL project aims at mapping the cold dust content of the inner Galactic disk, in a uniform, systematic way. Observations are performed using the Large APEX Bolometer Camera (LABOCA), installed at the APEX $12 \mathrm{~m}$ telescope ((Güsten et al. 2006)) on a $5100 \mathrm{~m}$ high site in Chile. LABOCA is composed of 295 bolometers operating at $870 \mu \mathrm{m}$ (Siringo et al. 2009). Our goals are to cover $\pm 60^{\circ}$ in Galactic longitude, over $\pm 1.5^{\circ}$ in latitude, to an r.m.s. of $50 \mathrm{mJy} /$ beam. For typical cold dusty clumps, at $1-10 \mathrm{kpc}$ distance, this corresponds to $5-\sigma$ detection limits of $\sim 1$ to a few $100 \mathrm{M}_{\odot}$.

Based on the first $95 \mathrm{deg}^{2}$ of data acquired in 2007, a preliminary catalogue of over 6000 compact sources brighter than $0.25 \mathrm{Jy} /$ beam could be extracted; about $30 \%$ of them can be associated with infrared sources from the MSX catalogue (Price et al. 2001), but most sources have no bright infrared counterparts. They could correspond to the earliest stages of (massive) star formation.

The observations should be completed by the end of 2009; the raw data will become public one year after each observing period. Advanced data products (calibrated maps, source catalogues) will also be made available upon completion. A detailed description of the survey and early results are presented in Schuller et al. (2009).

\section{References}

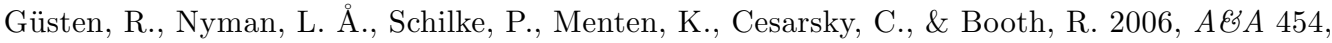
L13

Price, S. D., Egan, M. P., Carey, S. J, Mizuno, D. R., \& Kuchar, T. A. 2001, AJ 121, 2819

Schuller, F., Menten, K. M., Contreras, Y., et al. 2009, $A \& A$ in press

Siringo, G., Kreysa, E., Kovács, A., et al. 2009, A\& $\mathcal{E}$ 497, 945 